This item was submitted to Loughborough's Research Repository by the author.

Items in Figshare are protected by copyright, with all rights reserved, unless otherwise indicated.

\title{
Systems-based approach to investigate unsafe pedestrian behaviour at level crossings
}

PLEASE CITE THE PUBLISHED VERSION

http://dx.doi.org/10.1016/j.aap.2015.04.001

PUBLISHER

(C) Elsevier

VERSION

AM (Accepted Manuscript)

\section{PUBLISHER STATEMENT}

This work is made available according to the conditions of the Creative Commons Attribution-NonCommercialNoDerivatives 4.0 International (CC BY-NC-ND 4.0) licence. Full details of this licence are available at: https://creativecommons.org/licenses/by-nc-nd/4.0/

\section{LICENCE}

CC BY-NC-ND 4.0

\section{REPOSITORY RECORD}

Stefanova, Teodora, Jean-Marie Burkhardt, Ashleigh J. Filtness, Christian Wullems, Andry Rakotonirainy, and Patricia Delhomme. 2019. "Systems-based Approach to Investigate Unsafe Pedestrian Behaviour at Level Crossings”. figshare. https://hdl.handle.net/2134/20670. 


\section{Systems-based approach to investigate unsafe pedestrian behaviour at level crossings}

- Teodora Stefanova $a^{a, b,}$,

- Jean-Marie Burkhardt $t^{b}$

- Ashleigh Filtness ${ }^{a}$,

- Christian Wullems ${ }^{a}$,

- Andry Rakotonirainya,

- Patricia Delhomme

Highlights

- Gaps in the pedestrian behaviour at level crossings literature were identified.

-A new system-based framework (PULC) was proposed.

-PULC was applied to the analysis of focus groups data.

-Interacting factors contributing to crossing behaviour are presented graphically.

-Two AcciMaps illustrate a possible application of the PULC.

\section{Abstract}

Crashes at level crossings are a major issue worldwide. In Australia, as well as in other countries, the number of crashes with vehicles has declined in the past years, while the number of crashes involving pedestrians seems to have remained unchanged. A systematic review of research related to pedestrian behaviour highlighted a number of important scientific gaps in current knowledge. The complexity of such intersections imposes particular constraints to the understanding of pedestrians' crossing behaviour. A new systems-based framework, called Pedestrian Unsafe Level Crossing framework (PULC) was developed. The PULC organises contributing factors to crossing behaviour on different system levels as per the hierarchical classification of Jens Rasmussen's Framework for Risk Management. In addition, the framework adapts James Reason's classification to distinguish between different types of unsafe behaviour. The framework was developed as a tool for collection of generalizable data that could be used to predict current or future system failures or to identify aspects of the system that require further safety improvement. To give it an initial support, the PULC was applied to the analysis of qualitative data from focus groups discussions. A total number of 12 pedestrians who regularly crossed the same level crossing were asked about their daily experience and their observations of others' behaviour which allowed the extraction and classification of factors associated with errors and violations. Two case studies using 
Rasmussen's AcciMap technique are presented as an example of potential application of the framework. A discussion on the identified multiple risk contributing factors and their interactions is provided, in light of the benefits of applying a systems approach to the understanding of the origins of individual's behaviour. Potential actions towards safety improvement are discussed.

\section{Introduction}

\subsection{Level crossings are complex intersections}

Level crossings (LCs) are complex intersections where rail and road systems converge. At such intersections, road users are permitted to cross rail tracks when it is safe to do so (i.e. in the absence of an approaching train). There are two main categories of LCs according to the level of protection they provide to users. Passive LCs are equipped with static controls such as "STOP" or "GIVE WAY" road signs whilst active LCs, which are often riskier locations, are equipped with automatic controls such as red flashing lights or barriers. Based on feedback loops (top-down and bottom-up flow of information) between components, the ultimate objective of LCs' performance is to ensure road users' safe crossing through the rail tracks. At active LCs in particular, the system must provide enough and reliable information for the pedestrian to safely negotiate the crossing. Such information mainly consists of: raising the awareness of the crossing (e.g. LC approach signage); providing adequate physical characteristics of the crossing path (e.g. visibility, well defined LC quadrant); ensuring visibility and awareness of the warning controls and their purpose; raising awareness of the potential hazards at such crossings (e.g. risk of second train). Fatal crashes are more frequent at active LCs (characteristic of urban environments) than at passive (Australian Transport Council, 2010). Pedestrians are particularly vulnerable users of active LCs given the higher flow of pedestrian traffic in such areas (Cairney, 1992). Australia has widely deployed engineering interventions to improve pedestrian LC safety, such as automated pedestrian gates. However, despite such interventions pedestrian LC crashes still occur.

\subsection{Crashes at level crossings}

Despite a substantial decrease since the 1990s, the annual number of LC crashes worldwide remains unacceptably high (ATSB, 2012, Basacik et al., 2012, Evans, 2012 and Werkman et al., 2012). Although not as frequent as other types of road traffic crashes, they are associated with greater potential for fatal outcomes for victims and are related to serious economic costs (Evans, 2012, lorio et al., 2012 and Werkman et al., 2012). Not only do crashes at LCs impede on the operation and effectiveness of both rail and road infrastructure, but they also result in 
significant economic costs due to railroad property damage, insurance payments and legal fees (Iorio et al., 2012 and Metaxatos and Sriraj, 2012). In 2003, a cost per crash was estimated to range from $\$ 180,000$ (AUD) in urban areas to $\$ 430,000$ (AUD) in rural areas (Australian Transport Council, 2003). Data from the ATSB (2012) suggests that, similarly to data from the United States (Metaxatos and Sriraj, 2012), the number of crashes involving vehicles has noticeably declined in the last decades (i.e. between 2003 and 2007), whereas there has not been a significant change in the number of crashes with pedestrians.

\subsection{Pedestrian behaviour at LCs}

In a review of the literature examining the extent to which the systems approach has previously been applied to the investigation of a broad range of LC issues, Read et al. (2013) found that more than $70 \%$ of all existing publications on safety at LCs focused on the understanding and reduction of drivers' unsafe behaviour. Thus, only very limited information on the factors and conditions shaping pedestrians' unsafe behaviour at LCs is currently available. We subsequently carried out an in depth review of the literature with a strong focus on pedestrians' unsafe crossing. A number of keywords were used to identify publications relevant to: level crossings (i.e. level crossings; railway crossings; grade crossings; rail crossings); pedestrians (i.e. pedestrians; passengers; rail users; trespassers) and the rail industry more generally. The search was undertaken in the following electronic databases: Science Direct; EBSCOhost; Google and Google Scholar; HERDC (Higher Education Research Data Collection; Australia); and among researchers' network (conference proceedings and publications). Only 23 relevant publications; up to and including 2013 were identified. Four major gaps in the literature on pedestrian crossing at LCs emerged from the review of these papers (see Appendix A for detailed description):

The influence from research on motorist's behaviour.

Consistent with the large majority of the existing publications being on motorists, the review of the contributing factors applicable to pedestrian unsafe crossing have often been based on research on drivers' behaviour at LCs or on road safety publications more generally. Literature reviews underpinning past studies include only a small number of publications on pedestrians' behaviour at LCs. Moreover, there are publications which do not clearly report outcomes which apply to pedestrians versus motorists. The degree to which outcomes of driver focused literature can be inferred to pedestrians is unclear as the required skills and the corresponding constraints (e.g. legal, social) related to both types of users are different. 
The availability and quality of occurrence data.

In Australia and worldwide, the criteria for the classification of occurrence data are not always consistent between authorities and may include cases of suicide or trespass (i.e. walking across or along rail tracks at non designated crossing areas), which are known to have different precursors than transgressions at LCs (Evans, 2012 and Meiers et al., 2012). Thus, outcomes based on such data are hardly applicable between countries and even between regions. In addition, such data is associated with a limited range of identifiable risk factors, and struggles to comment on the cognitive or motivational origins of behaviour.

The lack of empirical research into the origins of unsafe behaviour. Instead of investigating the origins of unsafe crossing, studies often focus on providing frequencies of illegal behaviour or identifying high risk groups of users, and examine only a small number of key variables such as the observed reported efficacy and awareness of various controls (Basacik et al., 2012, Parker, 2002 and Stewart et al., 2004) or else the efficacy of education and enforcement campaigns (Lobb et al., 2001, Lobb et al., 2003 and Sposato et al., 2006). One study demonstrates pedestrians' likelihood of underestimating the speed of an approaching train as a result of a perception bias (Clark et al., 2013). Self-reported data from another study provides indication of the most relevant factors influencing decision-making of different types of users (Beanland et al., 2013).

The lack of research on multiple interacting risk-contributing factors.

Several authors have pointed to the advantages of investigating simultaneous interactions between multiple risk contributing factors as opposed to considering a single factors' contribution in isolation (lorio et al., 2012, Read et al., 2013 and Werkman et al., 2012).

High risk groups of users (i.e. young males) or times of the day (i.e. peak hours) have been predominantly associated with risky crossing along with a number of contributing factors such as: large groups of pedestrians, being in a hurry, inattention (distraction), sensation (thrill) seeking tendencies, status of the controls (closing vs. closed gates) or presence of a (visible) approaching train (Beanland et al., 2013, Clancy et al., 2007,Edquist et al., 2011, McPherson and Daff, 2005, Metaxatos and Sriraj, 2013, Searle et al., 2011 and Sposato et al., 2006). Davis Associated Limited (2005) are among the few who provided a classification of multiple factors influencing crossing behaviour, however they did not investigate the associations between different factors. In contrast, examining the interactions between various 
contributing factors Metaxatos and Sriraj (2013)showed that the presence and the larger number of pedestrian gates (i.e. at all LC quadrants) reduced the reported deliberate and observed (legal) violations independently of the train's direction. They also reported an increase in violations with the increasing number of pedestrians in a group (i.e. alone vs. in a group of two vs. in a group of more than two) independently of the time of the day. Finally, even though a number of authors have recently recognised the need to consider characteristics of the socio-economic area (e.g. presence of schools, industrial buildings) as a key factor shaping behaviour, to our knowledge such results have not yet been demonstrated (Edquist et al., 2011). While initial steps have been made to undertake research considering LCs as a complex system, these are rare and even rarer still is pedestrian focused systems research.

\subsection{Systems approach}

Systems theory, rooted in natural sciences has further been applied to the improvement of safety in complex systems. The systems approach aims to look at the problem as a result of the interaction of all the system's components, considering the whole as the unit of analysis, not just individual's behaviour. The utility of a systems approach in identifying factors which contribute to different types of errors has been demonstrated in a variety of complex systems such as aviation and, most recently, in the railway domain (Hobbs and Williamson, 2003, Li et al., 2008, Maurino et al., 1995, Rail Safety Regulatorś Panel, 2009 and Read et al., 2012). Read et al. (2012) were pioneers in applying a systems approach to the investigation of the associations between system factors and types of unsafe behaviour in rail incidents and accidents. However, they excluded occurrences at LCs from the scope of their analysis, given the added complexity of interactions between components of the road and the rail system.

With the fast pace of technological changes, there is a pressing urge to apply system models of safety and risk management to a broader range of domains (Hollnagel, 2004 and Leveson, 2004). So far, a number of models have been developed and predominantly have been applied to, or underpinned accident analysis within complex systems: Rasmussen's risk management framework and AcciMap (Rasmussen, 1997); Reason's Swiss Cheese Model (Reason, 1997 and Reason et al., 1990); Nancy Leveson's STAMP (Systems-Theoretic Accident Model and Processes, (Leveson, 2004); and the Safety through prevention and protection model (Hollnagel, 2004). While all of these models have emerged from the systems theory approach, each of them proposes a specific understanding of accident causation, investigation and prospective prevention. Traditional sequential and epidemiological models prioritise the analysis of the chain of events preceding an accident and are therefore considered inadequate to capture the dynamic nonlinear 
interactions between system components. Reason's Swiss Cheese Model (1990, 1997) for example, identifies latent system failures (e.g. inadequate procedures, decisions) which combined with active failures (errors) create conditions for accidents. However, it has been demonstrated that this model fails to explain how these factors are associated with the active failures and other system factors due to the static view of the state of the system (Johnson and Botting, 1999). In more recent models, accident causation is viewed as a complex network of nonlinear interactions between various levels of the system where decision-makers at higher system levels are considered as equally responsible for failures as frontline actors (Hollnagel, 2004 and Leveson, 2004). For example, Jens Rasmussen's AcciMap technique graphically illustrates the causal relationships between actions and decisions on higher system levels according to the information flow between different system components (Rasmussen et al., 2000). The risk contributing factors in an AcciMap are distributed over the six hierarchically organised system levels proposed in Rasmussen's risk management framework. Another example is the Nancy Leveson's STAMP approach seeking to explain accidents as failures to exercise control over concrete tasks contributing to the global system performance (i.e. safety constraints) (Leveson, 2004). The graphical representation of the STAMP technique is based on the identification of system constraints and causal factors associated with reasons for flawed control and dysfunctional interactions.

Independently of the applied method, the detection of causal factors implies an in depth description of the current state of the system's performance - a task implying serious methodological challenges. The system's performance is subject to constant changes related to time passage, scientific advances or the outcomes of past events and occurrences (Dekker et al., 2011 and Leveson, 2011). The more the systems performance is dynamic and variable, the more actors' behaviour becomes hard to predict. This is particularly true for open systems such as LCs - allowing interactions between systems' internal elements and the environment. Pedestrian crossing behaviour is influenced by the system's managing structures, by the actions and decisions of other independent actors (e.g. other pedestrians or motorists) and by a multitude of environmental characteristics (e.g. socio-economic and architectural characteristics of the urban and the narrower LC environment, weather and time conditions). Retrospective analysis of the relatively rare previous crashes is therefore limited in capturing risk factors at play in the specific circumstances and provides insufficient information about the current systems' state and performance. At the same time, prospective analysis is better placed to consider and predict systems' dynamics, although it would always be subject to changes over time and limitations related to the method of data collection and analysis (Dekker et al., 2011). 


\subsection{Aims of the paper}

This paper aims to define a new tool for the better understanding of pedestrian's behaviour accounting for the individual's interaction with other system components. The framework is supported by well-known theories in psychology and systems approach methods.

Given the infrequent nature of pedestrian crashes at LCs, the investigation of the links between precursors of behaviour and system factors would benefit from the use of prospective methods. In an effort to investigate the cognitive and motivational precursors of behaviour along with the contributing factors they are impacted by, a new system-based framework is proposed - "Pedestrian Unsafe Level Crossing framework" (PULC). In line with modern systems approach, this framework is also designed to be used in a prospective and predictive manner.

\section{Pedestrian Unsafe Level Crossing framework (PULC)}

\subsection{Origins and Structure}

The proposed PULC framework is inspired by two key sources: the Contributing Factors Framework developed for the classification of contributing factors to rail accident occurrences (Rail Safety Regulatorś Panel, 2009), and which is based on James Reason's Organisational accident causation model; and the Jens Rasmussen's risk management framework. Elements from both models have been combined to highlight potential links between specific risk factors identified in the existing literature and unsafe behaviour according to its level of intentionality. The PULC identifies factors on four system levels and is tailored to the particular context of pedestrian crossing behaviour (Fig. 1). 


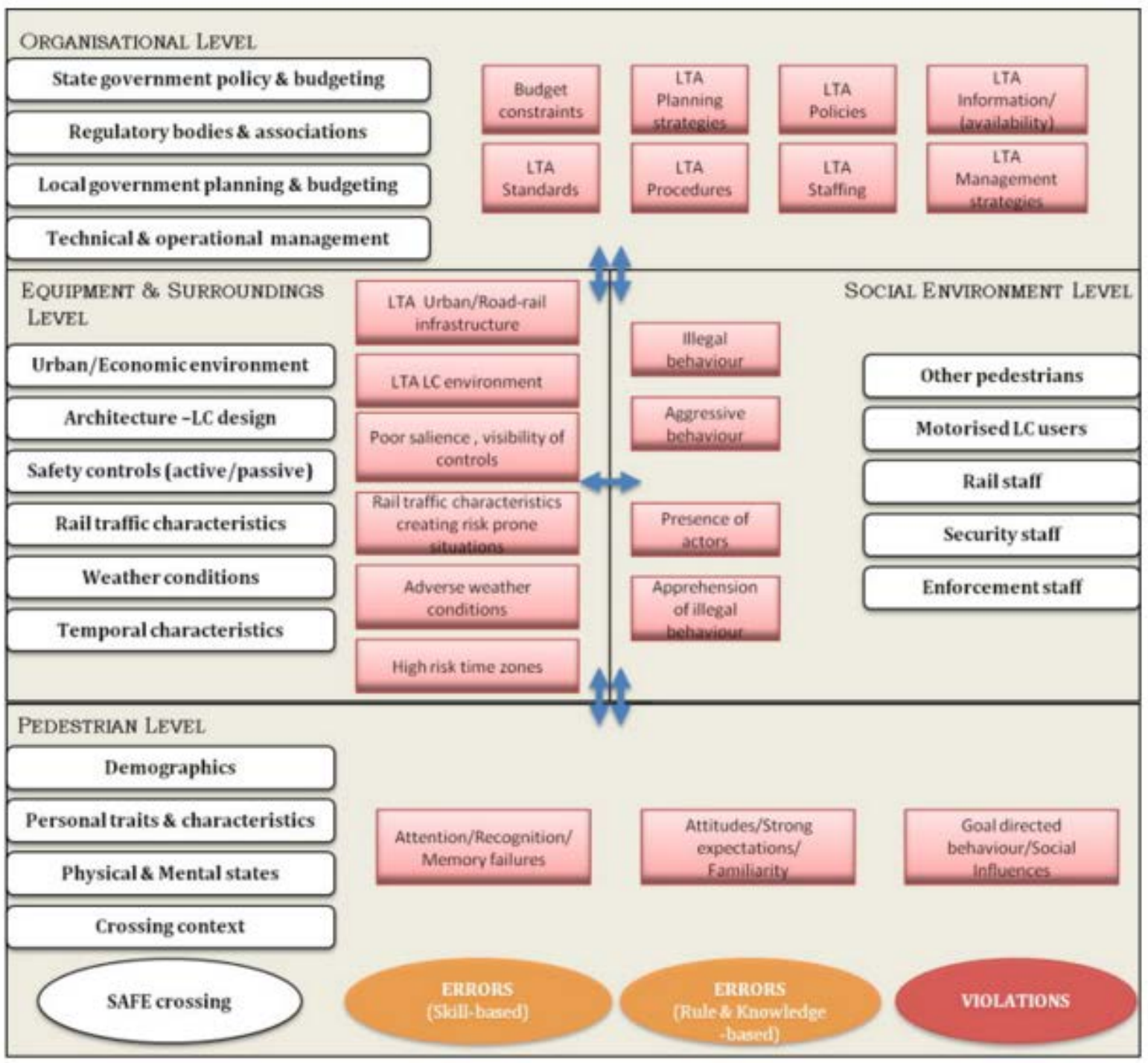

Legend:

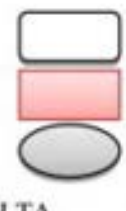

Components of the system levels

Risk contributing factors associated with the corresponding components

LTA

System performance outcome - crossing behaviour

Fig. 1.

Pedestrian Unsafe Level Crossing framework (PULC).

Each level lists system components responsible for various safety constraints associated with corresponding risk-factors potentially contributing to unsafe crossing. Unlike the proposed classification of system levels in Jens Rasmussen's model, in the PULC factors on the individual level (Pedestrian level) are distinguished from factors associated with the presence and behaviour of other actors in the immediate crossing context (Social environment). The Organisational and the Equipment and surroundings levels include components related to respectively systems' 
management and physical environment. Based on the current knowledge, components on each level have been identified in relation to their potential influence on pedestrian behaviour. The associated risk-factors are likely to have an impact on different types of unsafe behaviour.

We refer to James Reason's classification of "unsafe acts" which builds upon Jens Rasmussen's Skill, Rule and Knowledge based classification (SRK), to distinguish between errors and violations ( Reason et al., 1990). While errors result from failures on different levels of information processing (skill, rule, knowledge-based levels of performance) and are thus associated with cognitive precursors; violations emphasise on the role of social context in decision-making (e.g. social norms, rules, operating procedures) and are therefore associated with motivational factors which lead the person to intentionally deviate from the prescribed rules (Reason et al., 1990). According toParker et al. (1995) errors are more likely to be minimised through "retraining, redesign of the human-machine interface, memory aids, better information", whereas violations are more likely to be reduced by the modification of attitudes, norms, beliefs or the overall safety culture (p.1036).

The arrows in Fig. 1 represent the complex interactions between components within and between system levels. However, despite their interconnectedness various authors have demonstrated that system factors of different nature contribute to a larger extent to either errors or violations (Hobbs and Williamson, 2003 and Read et al., 2012).

Reason defines skill-based errors as actions which did not go as planned in automatic routine tasks requiring low attentional resources. In a LC context, such errors can be associated with internal or external distractions related to the perception or recognition of key elements in the LC environment (e.g. the activation of the automatic controls). Findings from previous studies have shown that the use of mobile devices while crossing alters pedestrians' vigilance and thus contributes to such errors (Clancy et al., 2007 and Metaxatos and Sriraj, 2013). Moreover, Hobbs and Williamson (2003)demonstrated that skill-based errors in aircraft maintenance are associated with factors related to the use of equipment, equipment malfunctions and environmental characteristics. Therefore, in the context of crossing at LCs, skillbased errors could be associated with risk-contributing factors on the Equipment and surroundings level. Such factors describe the characteristics of the LC environment (e.g. number of rail tracks, presence of controls), the larger urban area (e.g. key industrial and economic buildings within the area of the LC) or else, the temporal characteristics of the crossing moment (e.g. time of the day, weather conditions). Rule and knowledge based errors occur on the more conscious action-selection level. Rule-based errors occur in familiar situations when a person could misapply a good rule (e.g. assumptions: crossing is safe as long as there is not a visible train at active 
LCs) or apply a bad rule to a given situation (e.g. habits: crossing is perceived as safe as long as one train has passed even if the gates are not open). In contrast, knowledge-based errors describe failures related to a lack of information in novel situations. Thus, past experience (familiarity) would favour the formation of attitudes, strong expectations or habits likely to be associated with rule/knowledge based errors. In the context of crossing a LC, past experience would be associated with factors related to various laws, regulations and procedures governing the LC system (e.g. rail traffic management, enforcement, security, safety campaigns, road rules). In line with this assumption, Hobbs and Williamson (2003) demonstrated that risk factors associated with tasks requiring training, supervision, coordination or past experience with procedures were particularly associated with rule/knowledge based errors in aircraft maintenance. Within the PULC, factors related to the system's management are identified within the Organisational level, encompassing four higher system levels adopted from Jens Rasmussen's model.

Violations originate under the influence of various psycho-social constraints (e.g. attitudes, norms, beliefs) attributing a given value (importance) to the behaviour or to more abstract goals, and are generally carried out in the belief that they will result in more positive than negative outcomes. Reason (1990) defines violations as underpinned by risk/benefit trade-offs (e.g. I can take the risk to cross in order to catch the next train).

Goal attainment is a central concept in theories of self-regulation which explain individual's conscious efforts to influence thoughts, feelings and behaviours towards the achievement of goals in a dynamic environment (De Ridder and De Wit, 2006). While theories of self-regulation offer different perspectives, they share the idea that goals direct behaviour and give meaning to people's lives (Baumeister, 1989). Carver and Scheier (2001) propose a model of self-regulation rooted in the long tradition of expectancy-value theories in psychology explaining the persistence of efforts towards a given goal as driven by the perception of favourable outcomes. Such behaviour is defined in this paper as "goal directed behaviour". Having been widely applied in the domain of health and risk-taking, this model is based on the idea that goals differ in their level of abstraction and have a hierarchical structure. Goal directed behaviour can therefore explain risk-taking at LCs underpinned by the motivation of attaining higher level goals (e.g. "I need to violate the rules in order to be at work on time"). In this relation,Hobbs and Williamson (2003) demonstrated that time pressure was strongly associated with violations. In a LC context, Clancy et al. (2007) showed that $31 \%$ of survey pedestrians reported intentionally engaging in violations "to be on time" or to "catch a train". Violations can also be explained by social influences (i.e. crossing for a dare, to be part of the group). Various authors 
have shown that the larger number of pedestrians crossing together increases risktaking propensity at LCs ( Khattak and Luo, 2011 and Metaxatos and Sriraj, 2013). Nevertheless, deliberate violations can and often are underpinned by precursors of errors. In familiar situations decision-making and specifically risk/benefit trade-offs are often influenced by past knowledge and experience which potentially contribute to the formation of expectations, attitudes towards safety procedures or perception of risk (see examples of quotes in Appendix B related respectively to Safety checks, Safety campaigns and Unsafe behaviour).

\section{Applying the PULC - a focus group study with pedestrians}

Focus group discussions with pedestrians who frequently use LCs were organised. Participants were invited to share their past experiences at LCs, perception of risk and safety issues, opinions and observations of other pedestrians' behaviour. Such qualitative approach is a promising method to conduct explorative baseline studies on the identification of interactions between risk contributing factors within complex systems (Read et al., 2013 and Werkman et al., 2012).

In the next sections, the method of the study and some of the results are described to illustrate how the framework might contribute to a better understanding of the system's dynamics.

\subsection{Method}

\subsubsection{Participants}

Twelve participants between 16 and 75 years old $(m=42)$ took part in the focus groups. Two thirds of them were female (9/12). All were familiar with Wynnum Central LC. Participants reported also crossing LCs adjacent to Wynnum North train station $(N=3)$, Lindum train station $(N=1)$ and Cannon Hill train station $(N=1)$. All of these users were crossing from one to four times per day.

Four focus groups were formed as follows: one group of younger pedestrians (1630); two groups of middle aged (31-55) and, one group of older pedestrians (56+). The focus groups had on average three participants per group.

Participants were recruited via posters, inviting people who "regularly or occasionally walk through a level crossing" and are "over 15 years old". Posters were distributed at train stations and LCs within and nearby the Wynnum area (Queensland, Australia), as well as at large shopping centres in a close proximity. The rationale for targeting a sample from this area is that the majority of the reported near-misses with pedestrians from 2011 took place on this particular rail line (42\%), and specifically at the LC adjacent to Wynnum Central station (41\%) ( Queensland Rail, 2012). 


\subsubsection{Description of the frequented LCs by participants}

Wynnum Central is commonly frequented by all participants. It is equipped with active controls (flashing lights, barriers, a single pair of pedestrian lights and pedestrian gates on each side of the crossing) regulating pedestrian flow through the two rail tracks (Photograph 1). Some participants were also familiar with two other LCs (i.e. Cannon Hill and Lindum). These LCs differ from Wynnum Central as three rail tracks are separated by middle islands with active controls providing independent regulation of the pedestrian flow on each side.

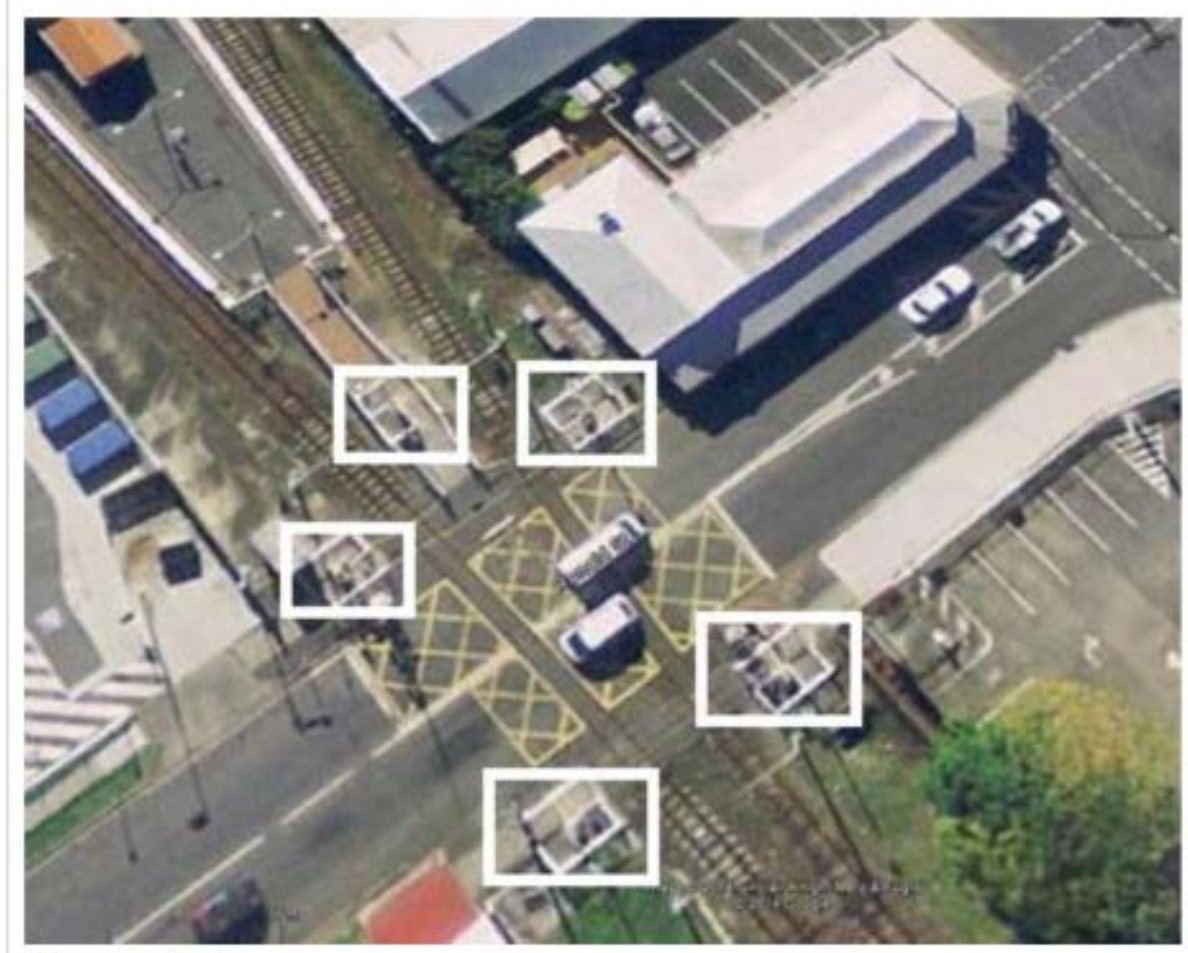

Photograph 1.

Wynnum Central level crossing.

Legend: Wynnum Central train station is loc ated on a middle island accessible through one of the two rail tracks of the LC. The station side of the crossing is operated by pedestrian gate system on each side of the road (indic ated with rectangles) and at the middle island. Unlike common design of other LCs giving access to train stations, during the activation of the controls pedestrian crossing is prohibited on both sides of the middle island irrespectively of the expected train's direction. On the opposite-station side, pedestrian gate systems are loc ated on each end of the pedestrian corridor. Each pedestrian gate system consists of an entry gate which closes automatic ally at the approach of a train and an emergency exit gate, which remains closed at all times but which could be pushed open from the inside of the LC if needed, or pulled open from outside the LC. (Source: Google earth 2014, eye altitude: $84 \mathrm{~m}$ ).

\subsubsection{Materials}

The focus groups were semi-structured utilising open-ended questions that covered six main themes incorporating all elements identified in the PULC (see Appendix B). The first theme consisted of introductory questions about participants' habitual 
crossing behaviour as a pedestrian (familiarity/frequency/purpose of crossing at concrete LC-site/s and knowledge about various aspects of crossing) and was simultaneously used as "breaking the ice" technique between participants. The remaining themes were discussed in each group in a non-systematic order. Each theme corresponded to a set of broad questions and probes developed beforehand to guide but not delimit the discussion. The second theme was developed to capture precursors of skill-based errors (e.g. absent minded behaviour, internal or external distractions, low vigilance). The third theme probed detailed description of unsafe crossing context and the explicitly provided causes (e.g. key elements of the environment, goal directed behaviour). Finally, the three last themes dealt with information on past experiences and attitudes, expectations and perceptions related to different aspects of: enforcement policies and procedures (e.g. likelihood to be penalised); education campaigns (e.g. exposure, perceived relevance, effectiveness); and general public safety awareness (e.g. perception of hazards or risks). It must be noted that because of ethical reasons, the last theme "Past occurrences at LCs" did not consist of pre-identified questions related to fatal occurrences.

\subsubsection{Procedure}

The aims of the study and privacy policies were explained to participants prior to the discussions. The sessions were conducted in a quiet environment, with groups of participants of similar age in order to enhance interactions and in particular the potential for sharing past illegal experiences (Yardley and Marks, 2004). Even though participants were asked to describe past crossing experiences they tended to talk more generally about their habitual safe or unsafe crossing. Probes based on five $\boldsymbol{W}$-questions ("Who-have you done that?"; "When - could you describe the situation?"; "What - what happened exactly?"; "Why- was there any particular reason?"; "Where - where did this happen?") were used to extend the discussion, provide clarifications and enhance the interaction between participants. Where possible (without interrupting the natural flow of the discussion), participants were asked to share their own knowledge, experience or opinions about topics raised by others. For example, if a pedestrian knew the exact amount of the sanction for illegal crossing - others were also explicitly asked. At the end of the discussion, all participants received an incentive for their time.

\subsection{Analysis}

Our analysis method was twofold. First, applying the PULC, factors potentially logically related to crossing behaviour and their interactions are identified. Second, 
two illustrative case studies of specific unsafe crossing occurrences are presented in AcciMaps format.

\subsubsection{Applying the PULC to identify and classify factors contributing to crossing behaviour}

\section{STEP 1. Identification of units of analysis - Cases}

Two researchers combined all the verbal exchanges that related to a depicted distinctive "Case" of crossing behaviour or elements likely to influence behaviour. For a case to be considered, even if it included the interaction between multiple participants, it had to meet one of three criteria:

Description of a crossing or events occurred at a specific time and place.

Description of aspects of habitual behaviour (e.g. crossing before the pedestrian gates are closed).

Description of general aspects of the system's properties (e.g. amount of the sanctions, existing safety campaigns) likely to influence behaviour.

\section{STEP 2. Categorisation of the profile of participants}

Participants' crossing profile was evaluated according to quantifiers identified in the verbatim informing on the frequency that a given behaviour was undertaken.

Pedestrians who did not report any previous unsafe crossing were assigned to "safe crossing profile". Error and violation profiles were assigned to pedestrians who reported at least one risky crossing experience. In this sample, all three participants who reported errors did not report any violations.

\section{STEP 3. Coding of factors potentially influencing crossing behaviour}

This coding was based on four variables:

Factor: any reference to a rationale, information or explanation regarding crossing behaviour or intentions. Two researchers used the pre-established categories of risk factors in the PULC as a first list for screening the case content. Multiple factors were identified within each case considering the wider context of the narratives. With the aim to detect a wide range of factors instead of quantifying their importance or prevalence, each was coded only 
once in relation to a given behaviour independently of the number of times or the number of participants it was mentioned by among all focus groups.

Actor (engaged in crossing): self (participant reporting own behaviour) vs. others (participant reporting observed behaviour).

Type of behaviour: safe crossing vs. error. vs. violation.

Nature of unsafe crossing: past experience (own, observed or information about an occurrence/behaviour) vs. hypothetical experience (expressed crossing likelihood for the self or others).

Factors identified within cases describing aspects of crossing (but not behaviour) were associated with safe behaviour, errors or violations according to the crossing profile of the participant. However, if for example a pedestrian assigned to error profile was talking about a hypothetical violation - then the factor was only associated with "hypothetical violation".

Finally each factor was associated with at least one and a maximum of ten different behaviours according to their type (safe vs. error vs. violation), their nature (past vs.hypothetical experience) and the actor (self vs. others).

\section{STEP 4. Classification of the factors within the pre-identified categories at each level of the PULC framework}

The researchers used a bottom-up process to group similar factors together and organised them in categories of risk factors corresponding to system components within the PULC framework. A more detailed list of risk factors were identified thus extending the proposed categories in the PULC. For example, factors related to "Active controls" and "Passive controls" were organised in separate categories as being likely to influence different cognitive and motivational precursors. While signs are likely to be overlooked, audible alarms or pedestrian gates' activation would more likely be misinterpreted than unnoticed.

\section{STEP 5. Coding of the interactions for each factor on the Pedestrian level with other system factors}

Finally, for each factor on the Pedestrian level one or two interactions (i.e. logical relationships) with other factors on the same or other system levels were identified. Only the system level of the identified interacting factors was taken into consideration (e.g. a factor within Goal directed behaviour category interacting with a factor on the Equipment and surroundings level). For each interaction, the number of 
behaviours accounting for the type, the actor and the nature of behaviour was counted. Three researchers participated in the coding process, discussed and resolved any discrepancies in opinions.

\subsubsection{Illustration of concrete relations between interacting factors and precursors of} behaviour utilising the AcciMap technique

To illustrate concrete relationships between precursors of behaviour and multiple factors on various system levels we adopted Jens Rasmussen's AcciMap technique, which has previously been applied to the investigation of the contributing factors to unintentional non-compliance at LCs (Salmon et al., 2013). The AcciMap analysis allowed representing the causal relationships between factors on the lower system levels and the implied contribution of factors from the higher Organisational level. The contribution of the latter was inferred as pedestrians were unlikely to provide information on organisational factors. Instead, exchanges with industry partners and rail experts allowed discerning governmental and industrial structures on each system level corresponding to the identified system components in the PULC framework. Thus, the inferred factors were assigned to each system level and category of factors in the AcciMap illustrations.

Each AcciMap included risk-factors within the four higher system levels as per Jens Rasmussen's model associated with specific organisational structures governing in Queensland and Brisbane area and likely to be responsible for the inferred riskcontributing factors. The typical Rasmussen's level "Physical processes and actor activities", which normally incorporates decisions and actions of frontline actors in the chain of events prior to the critical event (accident) was, for the purpose of this study and corresponding to the structure of the PULC, split into "Pedestrian level" and "Social environment level". Consistent with the adopted approach, this separation allowed the inclusive investigation of the origins of pedestrian behaviour taking into consideration influences from the surrounding social environment.

\subsection{Results}

Results are organised in three sections. First, descriptive statistics on the frequency and the types of unsafe behaviour are presented. Second, the classification of factors across system levels is presented followed by a discussion of the interacting factors associated with each type of behaviour. Finally, two case studies of violations are presented in AcciMaps format. 


\subsubsection{Reported unsafe behaviour and perceptions of high risk groups of users}

Eight out of the twelve participants mentioned at least one crossing experience which could be considered unsafe, the majority being reported by daily users $(N=6 / 8)$. Considering each age group, unsafe behaviour $(N=7 / 8)$ was mostly associated with younger (16-30 years old) and middle aged (31-55 years old) participants, while older adults (56+ years old) were most likely to report safe behaviour $(N=2 / 4)$.

Three crossing experiences associated with errors were identified in each age group of participants: one was inferred as such by the researchers and the others were recognised by the pedestrians after crossing. The three errors were classified as: a rule-based error (i.e. pushing the entry pedestrian gate while it had already started opening); a skill-based error (i.e. the participant failed to identify the activation of a second pair of pedestrian lights at a middle island); and a knowledge-based error (i.e. the participant was not aware of the presence of an emergency pedestrian gate during a first crossing experience in the country). In contrast, five younger and middle aged participants reported violations - deliberate crossing during active controls' activation, and being aware of the illegal nature of such behaviour.

Finally, within each participant's narrative we identified words and phrases describing other pedestrians as high at risk groups of users. All age groups of participants perceived younger pedestrians as high risk groups of users, even though some participants also suggested that school children tend to cross safely. Middle age participants were most likely to perceive older adults (elderly) as a high risk group, while only older adults did not perceive their age group as being at risk.

\subsubsection{The applied PULC}

After the coding, 298 factors were identified across all levels of the PULC. The Pedestrian level cumulated the largest number of identified factors distributed in 12 categories. The Environment and surroundings level comprised factors classified in 10 categories. The least number of factors were found on the Organisational and on the Social environment levels. Fig. 2 illustrates the distribution of factors within each category accounting for the number of factors associated at least once with safe crossing and/or error and/or violation (independently of whether the behaviour was hypothetical or past experience of the self or others). 


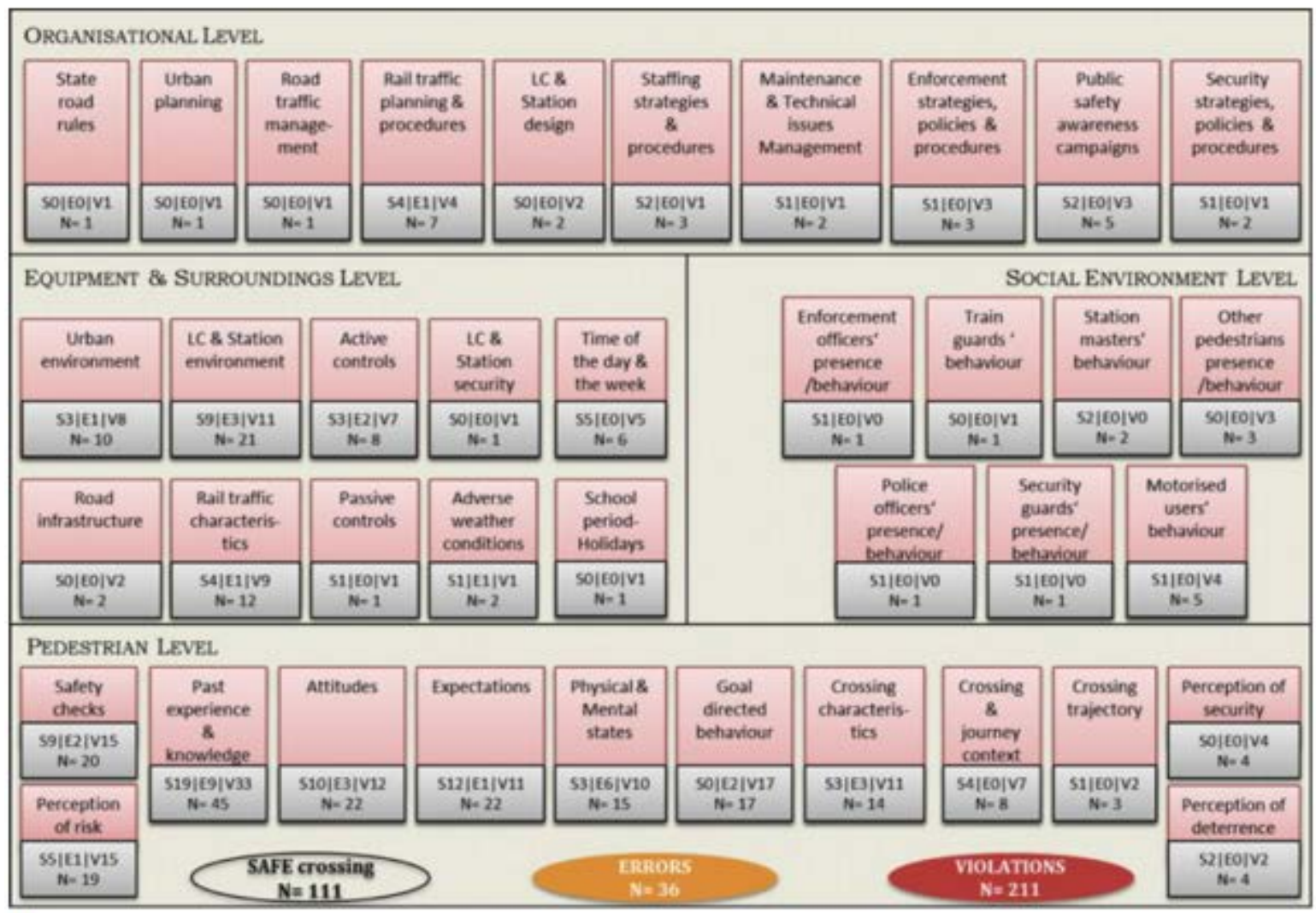

Fig. 2.

Applied PULC: detailed classific ation of contributing factors to pedestrian crossing behaviour.

Legend: For each c ategory of factors are provided: the number of factors associated with safe crossing

(S); the number of factors associated with errors $(E)$; the number of factors associated with violations (V) and last, the total number of factors identified in the category, noting that each factor could be associated with more than one type of behaviour.

"The majority of the identified factors on each system level were associated with violations and the least number - with errors" (Table 1). The Pedestrian level accounted for the largest number of identified factors. These factors were organised within categories corresponding to various cognitive and motivational precursors of behaviour. Factors within the Physical and mental states (e.g. impairments, emotions, moods),Crossing characteristics (e.g. crossing with earphones, carrying bags) and Safety checks categories were mostly associated with cognitive precursors of skill-based errors(11 out of 36). Factors within the Perception of risk, Attitudes, Past experience and knowledge and Expectations categories were mostly associated with cognitive precursors of rule/knowledge based errors (13 out of 36). Throughout the remainder of the analysis error types are considered together. Finally, factors within the Goal directed behaviour (e.g. time pressure, impatience with waiting times), Crossing and Journey context (e.g. going to work/school), Crossing trajectory (e.g. shortcuts, crossing on diagonal), Perception of deterrence and Perception of security categories were mostly associated with motivational precursors of violations. 


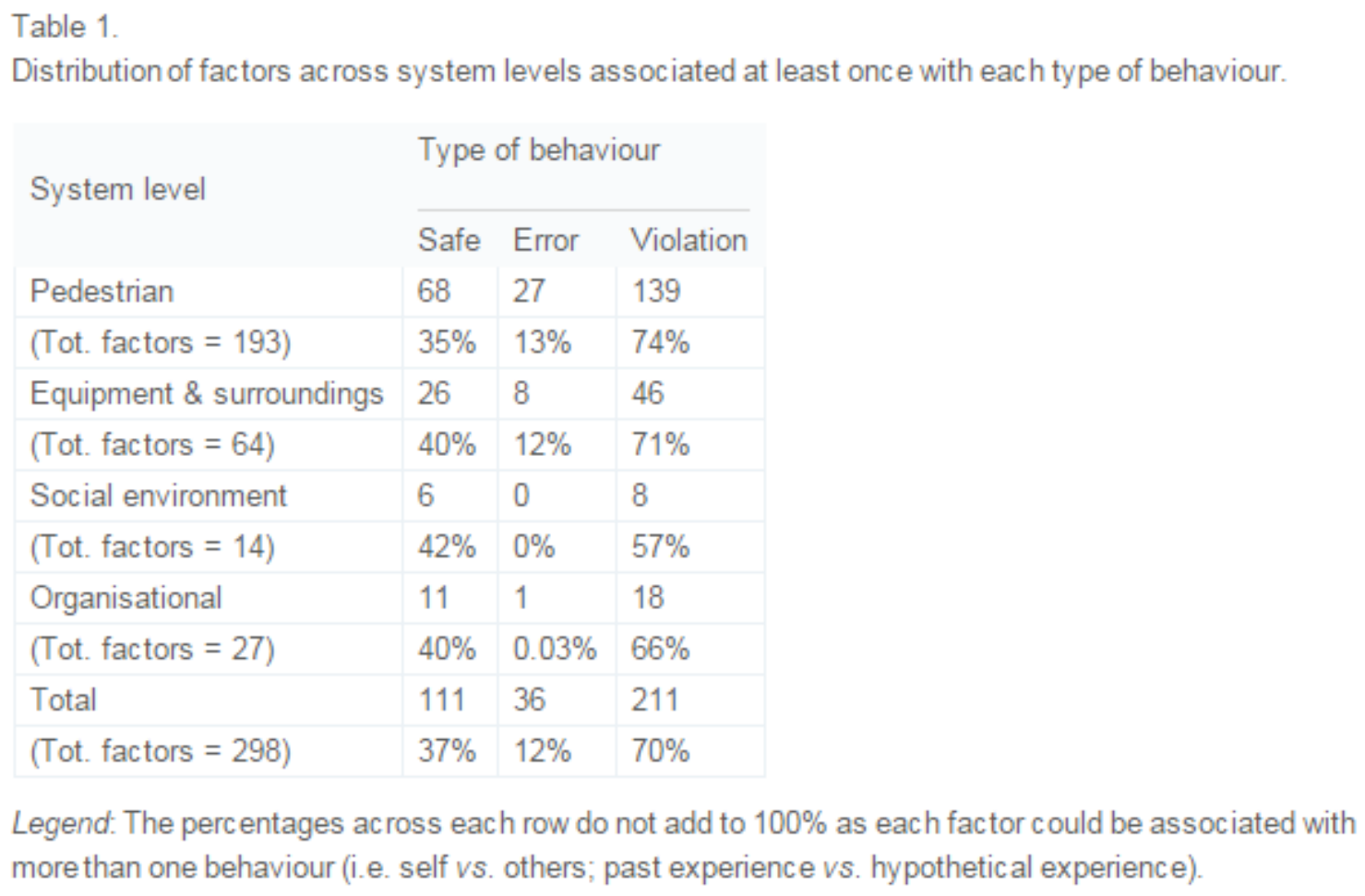

To further examine the influence of different system factors on the precursors of behaviour, in the following sections the interactions between factors within each category of the Pedestrian level and other factors on the same or other system levels are discussed in light of their association with either type of behaviour.

\subsubsection{Interactions between factors associated with safe crossing}

Among the 68 factors on the Pedestrian level associated with safe crossing (Table 1) five were found to interact with other factors on more than one system level. Thus a total number of 73 interactions associated with safe crossing were identified. Among these interactions 76 behaviours were counted, meaning that three of the interactions were associated with safe crossing reported both - for the self and for others.

Fig. 3 illustrates the contribution of interacting factors which were associated with safe crossing most often, independently of whether they were associated with the participants' own behaviour or with the behaviour of others. Among all categories on the Pedestrian level, the largest number of behaviours was associated with factors related toPast experience and knowledge, Expectations, Safety checks and Attitudes (68\%). The majority of factors associated with safe crossing interact with other factors on the Equipment and surroundings level (51\%) and to a smaller extent 
with factors on the Organisational and the Pedestrian levels. Influences from the social environment were least mentioned in relation to this type of behaviour.

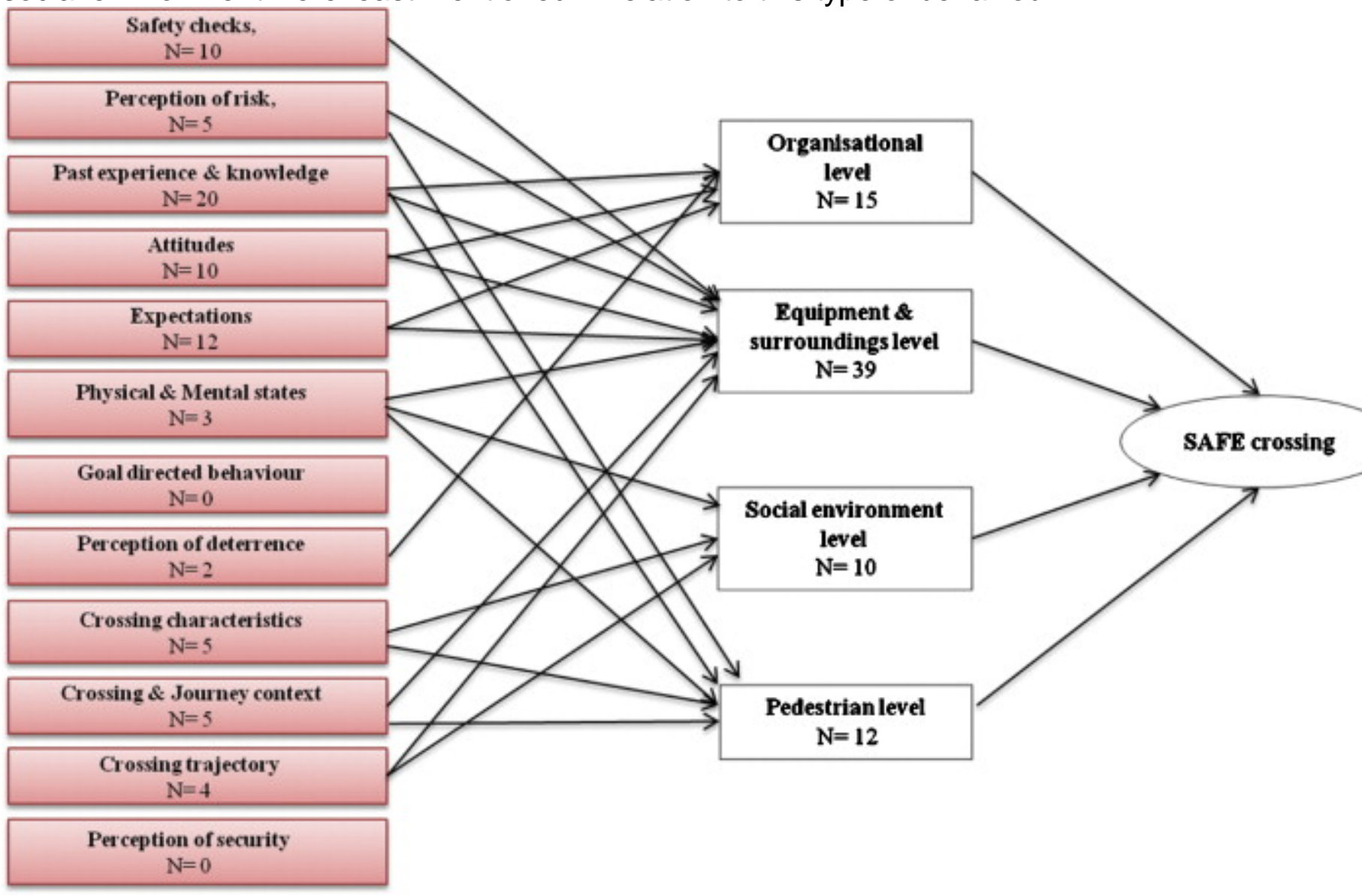

Fig. 3.

Illustration of interactions mostly associated with safe crossing.

Legend: Arrows illustrate the interactions most frequently associated with safe crossing;

$\mathrm{N}=$ the number of safe behaviours identified: (1) within each category of factors on the

Pedestrian level; (2) in interaction with other factors on each system level.

Factors on the Equipment and surroundings level were found to influence a large number of cognitive precursors of behaviour such as perception of risk, familiarity, attitudes and expectations but also motivational precursors. Among the cognitive precursors, knowledge about characteristics of the LC environment and rail traffic (e.g. the platform's height, the number of rail tracks, the angle or the distance to an approaching train) was often associated with an estimation of risk preceding the crossing decision (e.g. "If it was touch and go I wouldn't risk it"). Familiarity with active controls was associated with low likelihood of missing the controls' activation (e.g. the audible alarm can be heard before the gate system is active). In relation to this, participant's perception of risk appeared to be associated with checks for the status of the pedestrian gate or with checks for a visible approaching train. Among the motivational precursors, journey contexts were mentioned in relation to 
characteristics of the urban and the LC environment. For example safe crossing was associated with a goal of crossing the road instead of crossing to catch a train, but also with crossing in order to catch a train for leisure as well as for work.

Factors on the Organisational level seemed to contribute as well to the formation of attitudes and expectations and enhanced familiarity and knowledge about different aspects of the system's performance (cognitive precursors). For example, safe crossing was associated with: attitudes towards the amount of the sanction for illegal crossing; expectations based on others' experience with sanctions, expectations based on familiarity with train timetables; or else, awareness of previous occurrences or safety campaigns. Specifically, participants crossing safely expressed expectations that more express trains (not stopping at station) pass in the morning.

The influence of factors of the Social environment level mainly described crossing motivated by social influences. Specifically, safe crossing was associated with crossing with family (e.g. crossing with baby or with a partner) and crossing among random other pedestrians (e.g. not following other pedestrians). Moreover, crossing with known others was associated with more safety checks, whereas crossing among random others - with less. Finally, the interactions between factors within the Pedestrian level often described the relationship between past experience and perception of risk (i.e. knowledge about previous occurrences was associated with increased perception of risk).

\subsubsection{Interactions between factors associated with errors}

Among the 27 factors on the Pedestrian level associated with errors, four interacted with factors on two different system levels. Thus, in total 31 interactions were identified associated with 34 different behaviours. In other words, in three cases the same interaction was associated with more than one error. There were slightly more behaviours related to participants' own past experience or likelihood to commit an error (59\%) than were for others.

Fig. 4 illustrates the interactions between factors mostly associated with errors reported for the self (solid arrows) and with errors reported about others (dashed arrows). Among the categories of factors on the Pedestrian level, more than half of the identified errors (62\%) were distributed between Past experience and knowledge, Physical and mental states and Crossing characteristics. The largest percentage of factors associated with errors interacted with other factors on the Equipment and surroundings level (41\%) and the least percentage-with factors of the Organisational level (12\%). 


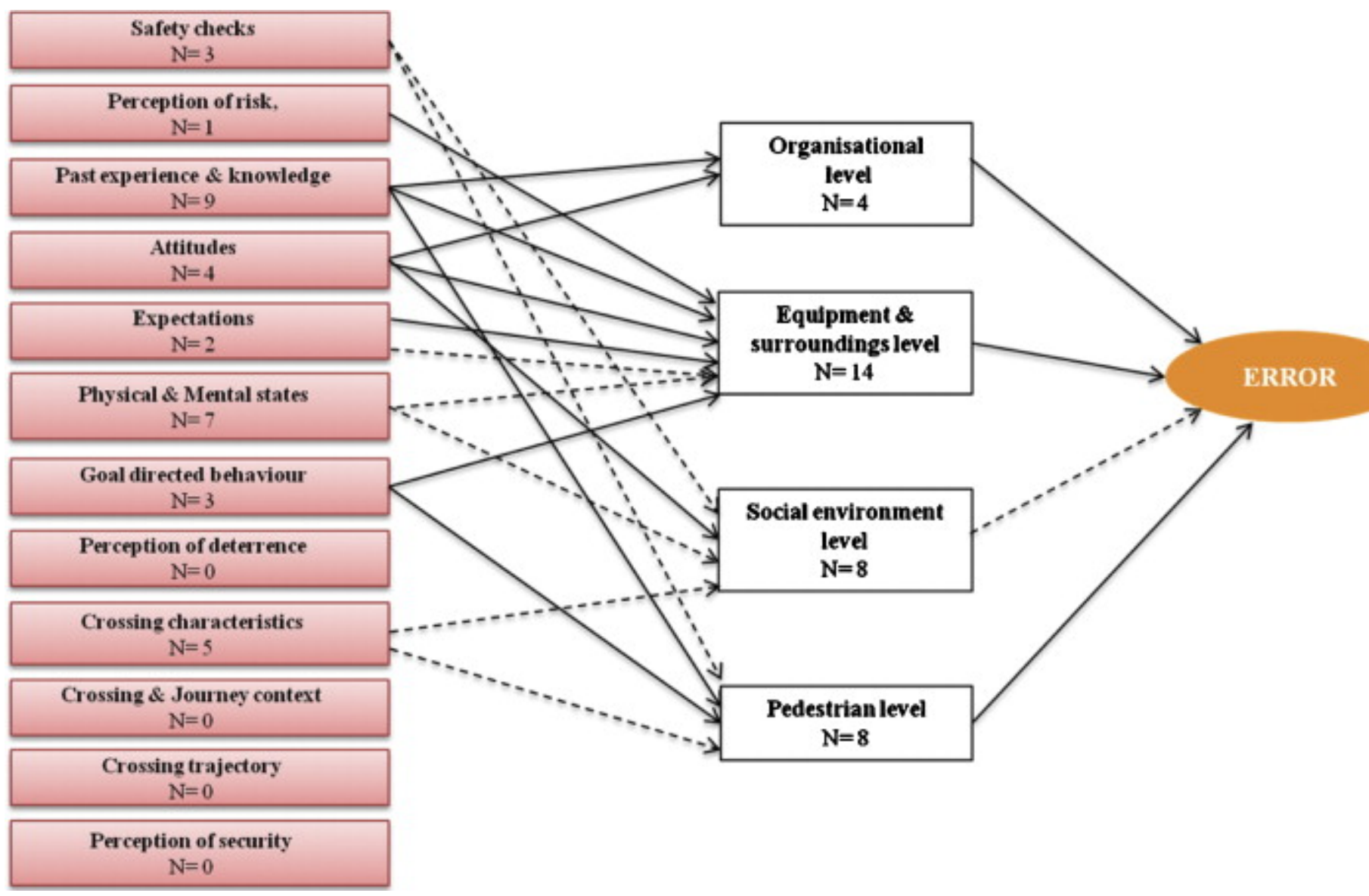

Fig. 4.

Illustration of interactions mostly associated with errors reported for the self and for other. Legend: Solid arrows correspond to interactions most frequently associated with participants' recognised errors or likelihood to commit errors; dashed arrows: correspond to interactions most frequently associated with knowledge or opinions about others' likelihood to commit errors: $\mathrm{N}=$ the number of errors identified: (1) within each category of factors on the Pedestrian level; (2) in interaction with other factors on each system level.

Factors associated with participants' previous recognised errors and perceived likelihood to commit an error, were mainly identified among the following categories of contributing factors: Past experience and knowledge, Attitudes and Goal directed behaviour and were interacting with other factors on the Organisational, the Pedestrian and the Equipment and surroundings levels. These interactions suggest that the likelihood of committing an error can be associated with less than adequate performance of rail staff (e.g. station masters do not act upon signalled problems) or else, with inadequate characteristics of the passive and active controls (e.g. frustration to wait for pedestrian gates to open, abundance of passive signs). The lack of previous experience at LCs, infrequent crossing and familiarity with more than 
one LC, were also associated with errors. Thus, the reported for the self past experience and likelihood to commit an error was associated with failures on the rule/knowledge based level of performance and even with goal directed behaviour. On the contrary, participants' perception of the likelihood of others to commit an error was predominantly associated with Physical and mental states and social influences, both hampering the capacity to detect the controls' activation (e.g.

hearing/visual/motor impairments, absent-minded behaviour, alcohol intoxication, crossing among a large group of pedestrians). Thus, unlike the factors associated with participants' own behaviour, reporting on others' behaviour was more likely to be associated with skill-based errors.

\subsubsection{Interactions between factors associated with violations}

The largest number of interactions $(N=165)$ was found for factors at the Pedestrian level associated with violations $(N=139$, Table 1$)$. Among all factors associated with violations, 26 were in interaction with other factors on two different system levels. Moreover, these interactions were mostly associated with more than one behaviour (i.e. reported past experience and/or hypothetical behaviour for the self and/or for others). In total 275 violations were identified, with the majority associated with participants' own reported behaviour (68\%, solid arrows) than with the observed or hypothetical behaviour of others (dashed arrows) (Fig. 5).

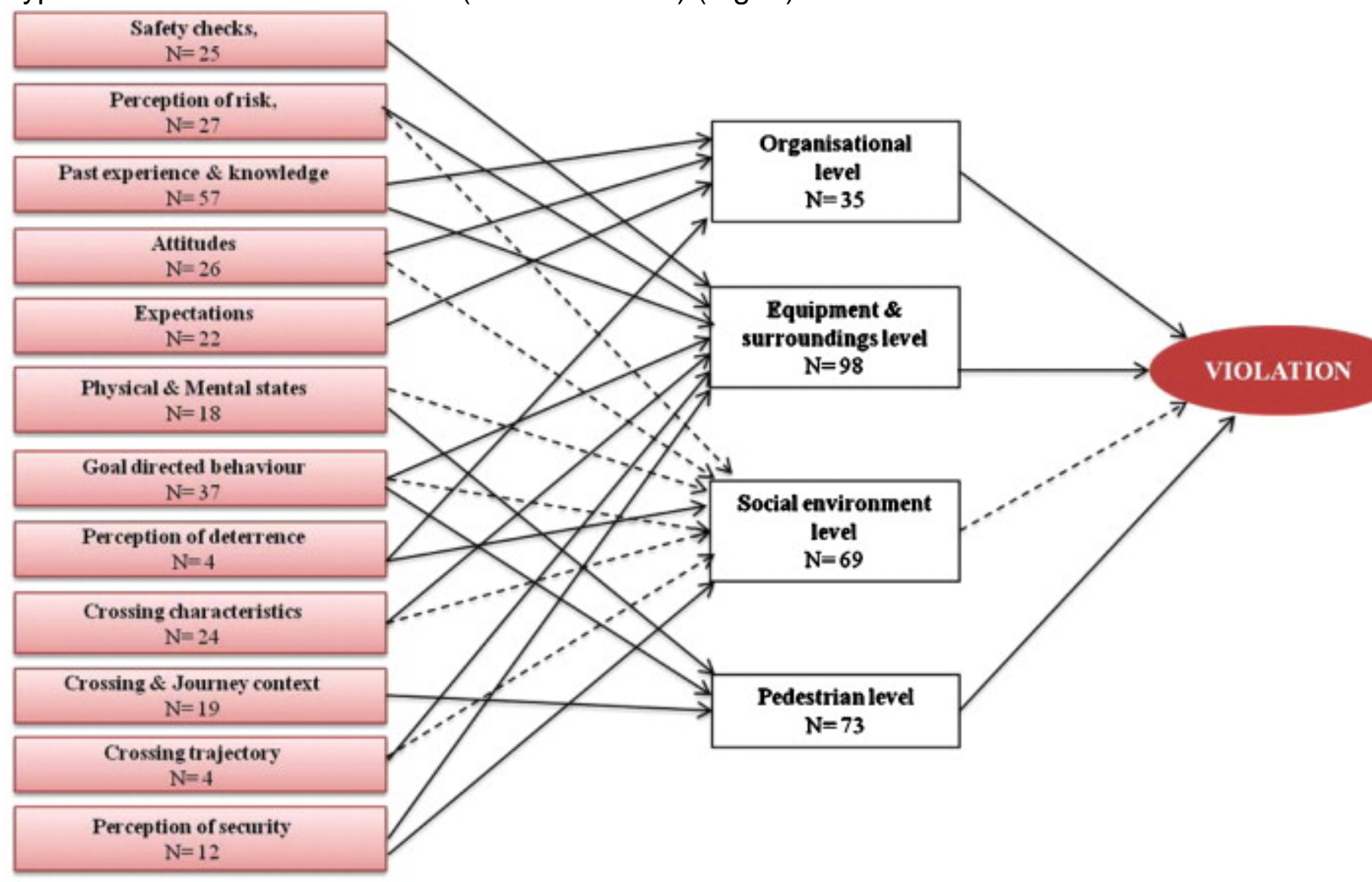


Fig. 5.

Illustration of interactions mostly associated with violations reported for the self and for others. Legend: Solid arrows correspond to interactions most frequently associated with participants' reported past experience or likelihood to commit violations. Dashed arrows correspond to interactions most frequently associated with knowledge or observed behaviour of others clearly intentionally violating or with the perceived likelihood of others to commit violations. $\mathrm{N}=$ the number of violations identified: (1) within each category of factors on the Pedestrian level; (2) in interaction with other factors on each system level.

Figure options

While the largest number of violations (21\%) was identified within the Past experience and knowledge category, factors within the following categories were associated with more violations per interaction: Goal directed behaviour, Crossing and Journey context, Crossing trajectory, Perception of deterrence and Perception of risk. Most violations, in total and per interaction, were associated with interacting factors on the Pedestrian and the Equipment and surroundings levels. Interacting factors on the Organisational level accounted for the least number of violations, however they were associated with more violations per interaction. On the contrary interacting factors on the Social environment level were associated with least behaviours per interaction although the total number of behaviours was higher. Violations reported for the self and associated with motivational precursors were identified within the following categories Goal directed behaviour, Crossing trajectoryand Crossing and journey context and were in interaction with other factors on the Pedestrian and the Equipment and surroundings levels. More than half of them concerned a reported likelihood to commit a violation (52\%) than past experience. These interactions described violations motivated by a fear of missing a train to (to work/school), unwillingness to wait for the lights at a nearby road traffic intersection, time pressure or impatience (e.g. shortcuts, diagonal). They were associated with various aspects of the LC and the larger urban environment, with rail traffic characteristics and with time and weather conditions. For example violations were associated with crossing after shopping, at any time of the day or out of peak hours, in the presence of a stopped train at station, or else when it's raining. Interactions with other factors on the Pedestrian level described goal directed behaviour underpinned by positive attitudes (e.g. "pushing the pedestrian [entry] gate open is quicker and more efficient").

Violations reported for the self were also associated with cognitive precursors corresponding to factors within the Past experience and knowledge, Attitudes and Expectations categories. These behaviours seemed to be influenced by other factors on the Organisational and the Equipment and surroundings levels. Logically they 
were more associated with past (71\%) than hypothetical experience. For example, violations were related to: awareness of the absence of locks on pedestrian entry gates, poor knowledge about the existence of pedestrian lights; awareness of second train arrival; familiarity with the platform, the larger area and with more than one LC. The large majority of the violations associated with low perception of risk (71\%) interacted with other factors of the Equipment and surroundings level (e.g. presence of stopped train, distance of approaching train, prior to closure of the pedestrian entry gate).

Various cognitive and motivational precursors were associated with violations reported as other pedestrians' observed or hypothetical behaviour, and were logically interacting with other factors of the Social environment level. These interactions described the perception of high risk groups of pedestrians. For example, they were associated with negative Attitudes (e.g. people are stupid, crossing for a dare is useless) or else, with a decreased Perception of risk compared to others' crossing behaviour (e.g. younger are unaware of the risk, younger are more at risk crossing for a dare, elderly with disabilities are more at risk, confident and people wearing high heels are more at risk). Contrary to participants' own behaviour, their perception of others' goal directed behaviour was associated with social influences and more specifically with sensation seeking tendencies (i.e. for a dare, to show up).

\subsection{AcciMaps of two case studies illustrating interactions between factors on} different system levels

Two crossing scenarios in which pedestrians reported violations at Wynnum Central LC were chosen because they involved a large number and different nature of factors from multiple system levels and were therefore considered as suitable to illustrate the potential use of the PULC.

\subsubsection{Case study 1}

The first case study identifies the risk contributing factors resulting in a violation against a closed pedestrian entry gate. AcciMap 1 (Fig. 6) shows the interaction between these factors playing a role in the decision, whilst being aware that an approaching train has been announced to pass through the LC. 


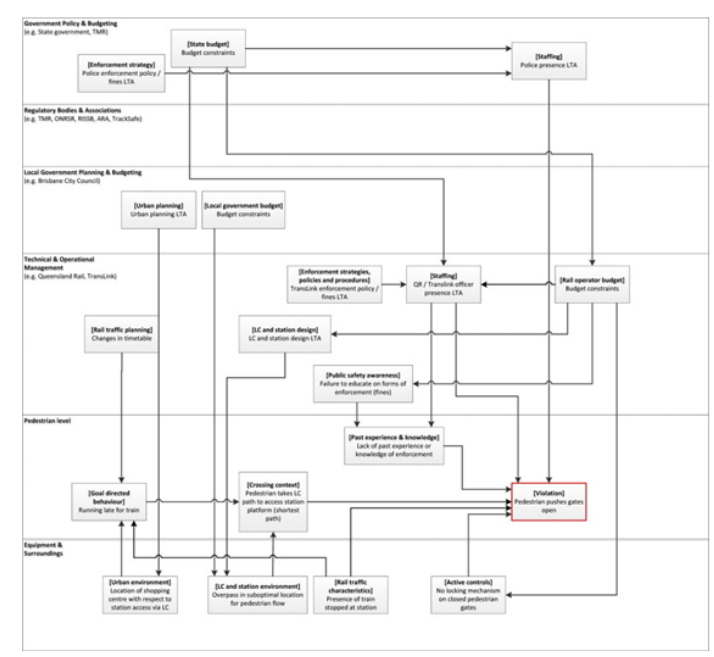

Fig. 6. Accimap 1 -illustration of crossing context where a pedestrian commits a violation on their way home after shopping.Legend LTA - less than adequate.

This particular crossing experience was reported by one participant - a 30-year-old female. She was on her way to Wynnum Central train station to catch a train home after shopping for groceries at a nearby supermarket. Evidence from the focus groups reveals that this is a commonly adopted trajectory by pedestrians, the LC being connected to a main road giving access to a nearby shopping centre, whereas an overpass is provided at the opposite LC and main road side end of the platform. Issues associated with the planning of the LC and overpass access points can therefore be inferred. According to the participant, her main reason for engaging in the particular crossing behaviour was the presence of a train already stopped at station. Crossing with her child, grocery bags, "school bag", "university bag", the participant ran to the platform, following the pedestrian path, she was unwilling to wait for the next train. As reported, she regularly crosses in identical situations ("I do it all the time...").

Such repetitive behaviour could be attributed to a low perception of risk or deterrence. Indeed, although not reported in relation to this particular crossing situation, the participant showed a low perception of risk associated with her familiarity with the LC environment: "I know the platform really well so if it was somewhere I haven't been before I couldn't gauge how far it (the approaching train) was if I didn't know how long the platform was" or with her (self-reported) "risktaking" tendencies ("I'm a risk taker"). A link was also established with pedestrians' reported lack of previous experience with sanctions: "see I do it all the time and I never got caught". This could be influenced by failures on the higher system levels to adequately plan or implement enforcement strategies (e.g. inadequate or limited enforcement staff presence or schedules). Queensland Rail - "QR" (Technical and organisational management level) for example shares the responsibility to provide 
enforcement with the State Police (State policy and budgeting level). Budgetary issues could impede on the appropriate implementation of enforcement strategies and procedures on both levels.

Participant's perception of risk can be also attributed to issues related to the design of the station platforms and the larger station environment (i.e. access to the platform through the LC). Moreover, the participant expressed positive attitudes related to the ease of pushing the pedestrian gates open (i.e. absence of locks):

"It's quicker and its more efficient just to push through the gates and run...its faster". Thus, the technical properties of the automatic controls might enhance pedestrians' intentions to violate. However, the implementation of new and more intricate controls would be constrained by the budget on the Technical and operational management level as well as on the State government level.

\subsubsection{Case study 2}

The second AcciMap (Fig. 7) represents a violation at the same Wynnum Central LC, which resulted in legal consequences for the participant. In this crossing situation, the pedestrian-a 55-year-old woman pushed the entry pedestrian gate open to catch a train on her way to work. The choice of trajectory (crossing the LC instead of taking the overpass) was here again a matter of the shortest path. However in this case, the pedestrian parked her car closer to the LC avoiding a car park (giving access to the overpass) where she had previously observed aggressive behaviour and therefore considered as unsafe location. 


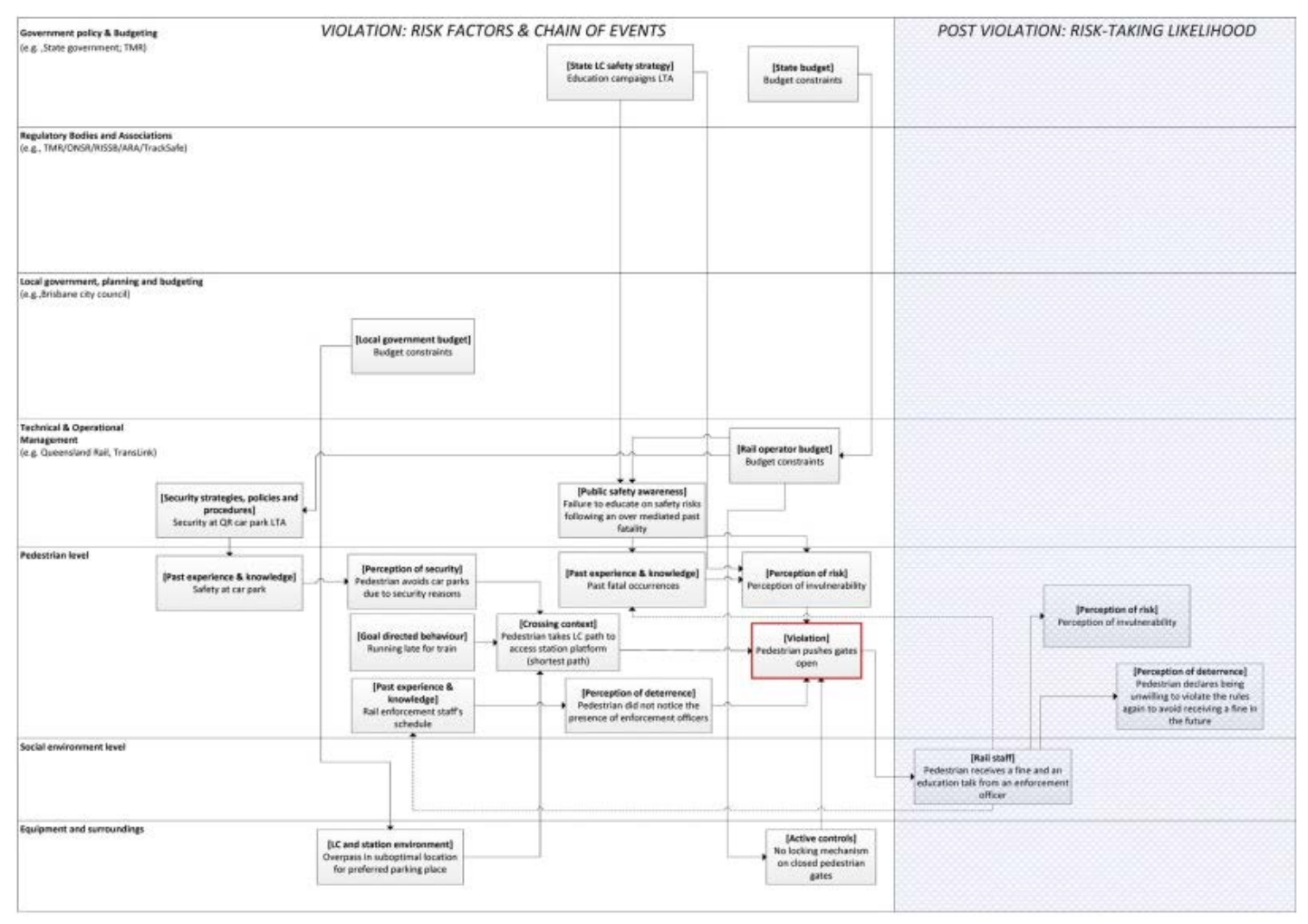

Fig. 7.

Accimap 2 - illustration of crossing context where a pedestrian receives a fine for a violation on their way to work.

Legend: LTA - less than adequate.

During the violation, she was issued a fine together with a young man crossing at the same time from the opposite side of the LC. Both pedestrians received the fines from undercover QR officers (transit officers). The main reported reason for crossing was the lack of locking mechanism on the pedestrian gates: "it's easy to open they don't lock the gate - it's easy to push and go". Here again, the technical configuration of the pedestrian gates was explicitly associated with increased likelihood to violate. The woman refers to pedestrian gates providing access to a different train station (Lota) which lock and cannot be opened, enhancing safe crossing. In addition to the ease of pushing the gate, the crossing decision in this situation could be linked to the expressed strong perception of control (e.g. "but I had time,see this is the danger, people are pushing the gate - so Lota they have a locking mechanism"). Similarly to Case study 1 , the perception of control in this situation could be explained by a low perception of risk or deterrence. However, in this case the pedestrian demonstrated a good knowledge about the regular presence of QR officers and associated this violation with a failure to recall this information (e.g. "I know, at 7.30 they are always there every 3 months... and I forgot that they are here... I haven't see them for a 
long time"), noting that the pedestrian recalled the exact time of when she was issued the fine (7.30 am).

In addition to exploring cognitive and motivational precursors of behaviour, Fig. 7 provides the opportunity to explore the extent to which they are influenced by the negative consequences of the action (i.e. receiving a fine). Specifically, the selfreported data post violation provides information on the effects of enforcement measures. The pedestrian reported a reinforced perception of deterrence (e.g. "I'll never do it again, I look at the station make sure they... I'll never do it again"), but also a maintained perception of control over the situation. In fact, according to QR policies, in addition to the sanction for illegal crossing transit officers are informing pedestrians on the risks of such behaviour. After having this discussion with enforcement officers, the pedestrian seemed to maintain a stronger perception of control than of deterrence.

"yes they told me that I shouldn't be doing this, you know, and it's dangerous, you know and, that I could be, you know killed and all that, but old people got killed cause they are slow-walking, or they can't hear. There was one lady she couldn't hear so the gate is very important it should be locked"

Arguably her perception of control was associated with a greater perception of risk for others than for herself in a similar situation (i.e. activated controls). She mentioned a specific past fatal occurrence at Wynnum Central train station 6 years prior to the focus group discussion. This collision was well known among multiple participants across focus groups, being widely covered by local media and discussed among community members (as indicated by participants). It appeared to have generated a lasting perception that elderly pedestrians are a high-risk group of LC users, due to their limited motor, visual or hearing capacities. It is worth noting that the exact reasons for this past occurrence are unknown ${ }^{1}$. Nevertheless, participants in the focus groups tended to explain the "old lady's fatality" in terms of impairments rather that statements or opinions as to whether she was aware or not of the risk she had taken (i.e. following a group of younger pedestrians pushing through the closed pedestrian gates). This is why it can be argued that this tragic past experience had more weight on the formation of crossing decisions than the educative discussion provided by enforcement officers.

\section{Discussion}

The conducted literature review on pedestrian crossing behaviour at LCs highlighted significant gaps in knowledge about the cognitive and motivational precursors of unsafe crossing and the associated multiple risk-contributing factors. To address these gaps, a system-based model called PULC (Pedestrian Unsafe Level Crossing framework) is proposed for the investigation of errors and violations at active LCs. 
This framework presents an extended view of various components of the system linked to cognitive and motivational precursors of behaviour (safe crossing, errors and violations). The PULC was applied to the analysis of qualitative data obtained from focus group discussions culminating in a detailed classification of factors derived from the descriptions of participants' safe and unsafe crossing. The classification facilitated the description of interactions between factors across system levels and moreover the examination of their role in the emergence of errors and violations. To our knowledge, this is the first study to provide an indication on the importance of groups of interacting factors according to their association with past and hypothetical violations reported for the self and for others.

Among the twelve focus groups participants, three had previously committed errors, five reported violations and the remaining four only declared safe crossing behaviour. Independently of the small number of participants, the broad discussions allowed information to be recorded relating to the observed crossing behaviour of others. The results presented here presented are used as an illustration of the application of the framework and cannot be considered as representative of the general population.

The analysis of the results on the interacting factors showed that factors on the Equipment and surroundings level are the most associated with each of the three types of crossing behaviour. Factors on the Organisational level were least associated with errors, whereas factors on the Social environment were least associated with safe crossing.

Safe crossing was more commonly adopted by older participants and infrequent users of LCs. It appeared to be predominantly associated with knowledge about previous near-miss or fatal occurrences. Safe crossing was also associated with cues in the environment impeding on the emergence of error-prone conditions (e.g. audible alarms) or influencing safe crossing decisions (e.g. train position, angle of visibility, tracks and platform characteristics). These results suggest that participants tend to perceive themselves as unlikely to commit errors, and moreover that their crossing decisions are rather associated with a perception of risk than with legal constraints. These results are consistent with previous findings, suggesting that pedestrians rely on the audible alarms to gauge a train's approach (i.e. controls' activation), but on the contrary - judge whether to cross according to the visibility of the approaching train (Beanland et al., 2013).

The identified errors were distributed evenly across all three age groups of participants. A similar number of factors were associated with errors referring to participants' own behaviour and to the behaviour of others. The small number of factors associated with errors is not surprising given that pedestrians might not be aware of their previous errors. Nevertheless, the findings allowed for the 
identification of potential failures of the system likely to create error-prone conditions. An identified rule-based error was associated with a participant pushing an opening entry gate to enter the LC after a train had passed not being aware of the illegal nature of such behaviour. In fact, according to information from pre-observations at this particular crossing site (Wynnum Central) and other LCs in Queensland, the precise opening moment and time required for the entry gate to fully open could vary among pedestrian gates at different LC angles. These technical characteristics of the automatic mechanisms might create conditions for rule-based errors, and influence pedestrians' perception of risk in a long term (i.e. expectations that the gate would take longer to start opening after a train had passed and thus pushing it open when it is still closed). Moreover, their trust in the equipment might be altered, especially at middle islands where pedestrian crossing is regulated separately on both sides of the island. They could also induce confusion and misinterpretation of the road rules if crossing seem to be prohibited at one LC angle and not at others due to discrepancies in the opening times. This result raises a question about the availability and the clarity of information about road rules for pedestrians. It underlines the importance of the homogeneity of the technical properties between active controls in the same geographical region. It also demonstrates the need for future similar and more in depth research specifically targeting the understanding of factors which lead to errors. In fact, most of the factors associated with errors reported for the self (rule-knowledge based) interacted with similar factors related to the technical and physical characteristics of active and passive controls and with familiarity with the LC environment. Therefore, arguably greater effort needs to be channelled towards improving the LC environment in a way that more information towards safe crossing is provided to users. In particular, more information about the purpose and the safe use of pedestrian gate systems (especially at LCs including middle islands) and presented in a more succinct manner (i.e. avoid abundance of passive signs) targeting the particular population of pedestrians (i.e. avoid confusions with road rules for motorists) would be likely to contribute to the reduction of errors. Finally, this result underlines the utility of the system approach to the detection of issues resulting from the dynamics of the LC system. Further research, should consider focusing on the investigation of the precursors of different types of errors.

Violations were most common with frequent LC users who were either younger or middle aged. The reported violations and likelihood of engaging in such behaviour were to a large extent associated with goal directed behaviour and were closely related to characteristics of the LC environment. A larger percentage of the violations associated with goal directed behaviour referred to hypothetical crossing, which 
could explain future risk-taking intentions (e.g. "I would cross if the train is far away"). Thus, risk-taking intentions appeared to be underpinned by previous unsafe experience associated with positive outcomes, and to be highly dependent on the perception of key elements in the physical environment.

The importance of aspects of the environment to the decision to violate was also illustrated in both case studies (AcciMaps). Specifically, the absence of locking mechanism on pedestrian gates could be a decisive element contributing to violations. While in past research, pedestrian gates were found to reduce unsafe crossing to a greater extent compared to other safety controls, it seems that the technical properties of such measures could play a crucial role to safety (Metaxatos and Sriraj, 2013). Arguably, there is a need to be sensitive to contextual differences across jurisdictions when interpreting other research. In relation to this, the Applied PULC framework controls for a rather exhaustive list of factors if the same behaviour is to be compared between two different populations and/or territories.

In addition to goal directed behaviour, reports of one's own violations were also associated with attitudes, knowledge and expectations related to different aspects of organisational factors such as safety campaigns or enforcement procedures. Such interacting factors were often related to knowledge about others' behaviour associated with low perception of risk with regards to own behaviour (e.g. "younger pedestrians only receive warning", so I am not at risk) as illustrated in the second case study (AcciMap 2). Thus, violations underpinned by precursors of rule/knowledge based errors and based on the observation of others' behaviour could potentially be mitigated through actions at the Organisational level targeting the improvement of various procedures, and strategies (i.e. changes in enforcement procedures, rail staff training, and safety campaigns targeting specific age groups of pedestrians).

The presence and behaviour of other pedestrians was not associated with selfreported violations. However, in line with past research, crossing among others was associated with decreased safety checks. Violations among a large number of pedestrians could be explained by an increased distraction or by a "diffusion of responsibility" - a psychological phenomenon rooted in the theories of social influences describing the delegation of responsibility in the presence of others executing the same task. Explaining taking risks in road safety, the diffusion of responsibility has been demonstrated byHarrell (1991) who found that pedestrians' cautiousness is reduced in the presence of others crossing on the opposite road side. In this relation, a clear intention to violate in the presence of others in order to avoid being issued a fine was reported by one of the participants. Similarly, as illustrated in the second case study (AcciMap 2), the presence of another pedestrian crossing at 
the same moment might have influenced the crossing decision, provided that the participant expressed a perception of control after the infringement. Moreover, the participant expressed future intentions to look for enforcement officers before crossing, such statement being likely to explain a strategy to avoid violations only in the presence of enforcement officers. This finding highlights a potential need to adopt strategies towards the improvement of the working schedules of enforcement staff (i.e. avoiding repetitive appearance).

Considering factors associated with the perception of the crossing behaviour of others, it could be argued that the large majority are influenced by a low perception of risk for the self compared to others. Unlike the reported errors in participants' own behaviour, the perception of others' likelihood to commit errors was associated with social influences (e.g. "following others blindly"). Similarly, the observed violations of other pedestrians were associated with negative attitudes and a perception of more at risk groups of users than the self, independently of whether it corresponded to their own age group or not. Such phenomenon in social psychology is known as "illusion of invulnerability" (i.e. comparative optimism, optimistic bias, unrealistic optimism) and refers to people's tendency to perceive themselves as less likely to experience negative events than others (Harris and Middleton, 1994). Illusion of invulnerability could be explained by the perception of control and low perception of risk and has been associated with various types of risk-taking (i.e. smoking, health checks). Thus, to reduce such bias in the perception of the risk of crossing, the origins of the bias need to be further investigated in order to develop more effective safety measures.

\section{Conclusion, limits and perspectives}

This analysis and the results confirmed that focus group transcripts can be used to identify contributory factors of behaviour and in particular are a rich source of data pertaining to the lower system levels, which could be used to infer failures or necessary actions towards safety improvement to be taken on higher system levels. It is possible that the lack of higher level factors is an artefact of the focus group participants being non-rail expert end users of the LC. Future studies may wish to consider applying a similar technique to focus group discussions with rail employees and experts from influential organisations at the higher system levels.

The factors associated with violations might be subject to a social desirability bias, omissions or a poor recall of the exact conditions under which the behaviour took place or the motivations that underpinned it. Even though in this data set participants logically reported factors influencing their recent or future behaviour, such evidence cannot be considered as describing the "current" state of the system because of its 
permanently changing and dynamic nature. However, such limitations are to a point compensated by the benefits of looking into multiple factors' contribution to crossing behaviour, a hardly attainable objective by traditional methods such as crash investigation for example.

In addition, the presentation of two case studies of unsafe crossing behaviour demonstrated how factors from different levels of the system interact to influence pedestrian crossing behaviour. This novel application of the AcciMap technique (Rasmussen, 1997) provided new insights into potential areas of weakness within the LC system which facilitate unsafe behaviour. For example, pedestrians' decision to access the station from the LC seemed to be potentially correlated to inadequate planning of the station design considering the larger urban environment (i.e. location of overpass; security issues or convenience with regards to journey context). Future efforts could be concentrated on the redesign of the station environment and particularly to solutions providing pedestrians with direct (path of least resistance) access points to the LC.

In addition, the use of AcciMaps demonstrated wider applications for this technique beyond traditional accident analysis. As shown by the current analysis, the same behaviour is likely to be underpinned by various precursors requiring different remedial measures. Therefore, AcciMaps linking cognitive and motivational precursors of behaviour to actions and decisions of frontline actors or higher level decision-makers would allow more specific description of (potential) issues and failures within the system and thus facilitate the identification of more specific actions towards safety improvement.

The PULC could be further applied to the analysis of larger data sources allowing specialists from a wider range of areas (e.g. experts in social, engineering, marketing sciences) to collaborate towards the prevention of future incidents and the prediction of future problems. For example, this may be achieved by the development of simulation studies with the aim to predict and test the efficacy and/or potential issues of new safety measures taking into consideration the identified and inferred risk contributing factors.

In conclusion, the current study provides evidence that pedestrian crossing behaviour at LCs is influenced by a wide range of factors from across the rail LC system. This cross system level influence on behaviour is in line with previous research at rail LCs (Read et al., 2013) and wider accident analysis (Rasmussen, 1997 and Reason, 2008). The presented PULC framework provides new insight into explaining why unsafe acts are undertaken by pedestrians at LCs and can be used to propose more integrative future interventions to mitigate unsafe crossing behaviour. 


\section{Acknowledgements}

The authors are grateful to the CRC for Rail Innovation (established and supported under the Australian Government's Cooperative Research Centres program) for the funding of this research. Project No. R2.120: Understanding Pedestrian Behaviour at Level Crossings. The authors wish to thank the anonymous reviewer whose comments have improved this paper.

\section{References}

ATSB., 2012. Australian rail Safety Occurrence Data 1 July 2002-30 June 2012, Australian Rail Safety Occurrence Data (pp. 48). Canberra: Australian Transport Safety Bureau.

Australian Transport Council, 2003. National Railway Level Crossing Strategy. Canberra, Australia.

Australian Transport Council, 2010. National Railway Level Crossing Strategy 2010-2020.

D. Basacik, S. Cynk, T. FlintSpotting the signs: situation awareness at level crossings Paper presented at the Global Level Crossing and Trespass Symposium, London, UK (2012)

R.F. BaumeisterThe optimal margin of illusion J. Social Clin. Psychol., 8 (1989), pp. 176-189

V. Beanland, M.G. Lenné, P.M. Salmon, Stanton, NAA self-reported study of factors influencing decision-making at rail level crossings: Comparing car drivers, motorcyclists, cyclists and pedestriansPaper presented at the Australian Road Safety Research, Policing \& Education Conference, Brisbane, Queensland (2013)

P. Cairney Improving safety at railway level crossings: Adelaide Convention Centre, Australia, 26-27 September 199 Appl. Ergon., 23 (5) (1992), pp. 363-365 http://dx.doi.org/10.1016/00036870(92)90360-8

C.S. Carver, M.F. Scheier On the Self-Regulation of Behavior Cambridge University Press (2001)

Clancy, J., Dickinson, S., Scott, M., 2007. Study of pedestrian behaviour at public railway crossings (D. o. Infrastructure, Trans.): Lloyd's Register Rail Limited.

H.E. Clark, J.A. Perrone, R.B. Isler An illusory size-speed bias and railway crossing collisions Accid. Anal. Prev., 55 (0) (2013), pp. 226-231 http://dx.doi.org/10.1016/j.aap.2013.02.037

Davis Associated Limited, 2005. Level crossings. Summary of findings and key human factors issues. 
D. De Ridder, J. De Wit Self-regulation in Health Behavior: Concepts, Theories, and Central Issues Self-regulation in Health Behavior John Wiley \& Sons Ltd., Chichester (2006) pp. 264

S. Dekker, P. Cilliers, J.-H. Hofmeyr The complexity of failure: Implications of complexity theory for safety investigations Saf. Sci., 49 (6) (2011), pp. 939-

945 http://dx.doi.org/10.1016/j.ssci.2011.01.008

Edquist, J., Hughes, B., Rudin-Brown, C.M., 2011. Pedestrian non compliance at level crossing gates CURTIN-Monash accident research centre.

Evans, A.W., 2012. The economics of railway safety. Research in Transportation Economics(0). http://dx.doi.org/10.1016/j.retrec.2012.12.003.

W.A. Harrell Factors influencing pedestrian cautiousness in crossing streets J. Social Psychol., 131 (3) (1991), pp. 367-372 http://dx.doi.org/10.1080/00224545.1991.9713863

P. Harris, W. Middleton The illusion of control and optimism about health: on being less at risk but no more in control than others Br. J. Social Psychol., 33 (4) (1994), pp. 369386 http://dx.doi.org/10.1111/j. 2044-8309.1994.tb01035.x

A. Hobbs, A. Williamson Associations between errors and contributing factors in aircraft maintenance Hum. Factors: J. Hum. Factors Ergon. Soc., 45 (2) (2003), pp. 186201http://dx.doi.org/10.1518/hfes.45.2.186.27244

E. Hollnagel Barriers and Accident Prevention Ashgate, Burlington. VT (2004)

L. Iorio, S. De Marco, E. Cosciotti Life momentum at level crossing: Human factor, road-rail safety policies, available technologies. A cross-sectorial challenge playing a rewarding role for the upgrade of safer mobility options Paper presented at the Global Level Crossing and Trespass Symposium 2012, London, UK (2012)

C.W. Johnson, R.M. Botting Using reason's model of organisational accidents in formalising accident reports Cognit. Technol. Work, 1 (2) (1999) http://dx.doi.org/10.1007/s101110050037

A. Khattak, Z. Luo Pedestrian and bicyclist violations at highway-rail grade crossings Transp. Res. Rec.: J. Transp. Res. Board, 2250 (1) (2011), pp. 76-82 http://dx.doi.org/10.3141/2250-10

N. Leveson A new accident model for engineering safer systems Saf. Sci., 42 (4) (2004), pp. 237-270 http://dx.doi.org/10.1016/S0925-7535(03)47-X 
N. Leveson Applying systems thinking to analyze and learn from events Saf. Sci., 49 (1) (2011), pp. 55-64 http://dx.doi.org/10.1016/j.ssci.2009.12.021

W.-C. Li, D. Harris, C.-S. Yu Routes to failure: analysis of $\mathbf{4 1}$ civil aviation accidents from the Republic of China using the human factors analysis and classification system Accid. Anal. Prev., 40 (2) (2008), pp. 426-434 http://dx.doi.org/10.1016/j.aap.2007.07.011

B. Lobb, N. Harre, T. Suddendorf An evaluation of a suburban railway pedestrian crossing safety programme Accid. Anal. Prev., 33 (2) (2001), pp. 157-165 http://dx.doi.org/10.1016/s00014575(00)00026-9

B. Lobb, N. Harré, N. Terry An evaluation of four types of railway pedestrian crossing safety intervention Accid. Anal. Prev., 35 (4) (2003), pp. 487-494 http://dx.doi.org/10.1016/s00014575(02)00026-x

D.E. Maurino, J. Reason, N. Johnston, R.B. LeeBeyond Aviation Human Factors Ashgate (1995)

C. McPherson, M. Daff Pedestrian behaviour and the design of accessible rail crossings Paper presented at the 28th Australian Transport Research Forum, Sydney, Australia (2005)

S. Meiers, H. Guo, M. Levasseur Crossing, vehicle and environmental characteristics influence on crash likelihood in Australia and New Zealand Paper presented at the Global Level Crossing and Trespass Symposium (2012)

P. Metaxatos, P.S. Sriraj Advancing pedestrian safety at rail grade crossings Transp. Res. Rec. (2012)

Metaxatos, P., Sriraj, P.S., 2013. Pedestrian/Bicyclist Warning Devices and Signs at Highway-Rail and Pathway-Rail Grade Crossings Civil Enginneering Studies: Illinois Center for Transportation Series.

A. ParkerSecond train coming warning sign demonstration projectsTransp. Res. Board Natl. Acad. (2002)

A. Parker, J. Reason, A. Manstead, S. Stradling Driving errors, driving violations and accident involvement Ergonomics, 38 (5) (1995), pp. 1036-1048

Queensland Rail., 2012. Level crossings near miss table (2011). http://www.queenslandrail.com.au/Safety/Pages/Levelcrossings.aspx 
Rail Safety Regulatorś Panel., 2009. Contributing Factors Framework Manual. Fortitude Valley, Queensland 4006: Rail Safety Regulators' Panel.

Rasmussen, J., 1997. Risk management in a dynamic society: a modelling problem. Safety Science, 27(2-3), 183-213. http://dx.doi.org/10.1016/S0925-7535(97)00052-0.

Rasmussen, J., Svedung, R., Svedung, I., 2000. Proactive Risk Management in a Dynamic Society: Swedish Rescue Services Agency.

G.J.M. Read, M.G. Lenné, S.A. Moss Associations between task, training and social environmental factors and error types involved in rail incidents and accidents Accid. Anal. Prev., 48 (0) (2012), pp. 416-422 http://dx.doi.org/10.1016/j.aap.2012.02.014

Read, G.J.M., Salmon, P.M., Lenné, M.G., 2013. Sounding the warning bells: The need for a systems approach to understanding behaviour at rail level crossings. Applied Ergonomics(0). http://dx.doi.org/10.1016/j.apergo.2013.01.007.

J. Reason Human Error Cambridge University Press. (1990)

J. Reason Managing the risks of organizational accidents Ashgate (1997)

J. Reason The human contribution: unsafe acts, accidents and heroic recoveries. Farnham [etc.] Ashgate (2008)

J. Reason, A. Manstead, S. Stradling, J. Baxter, K. Campbell Errors and violations on the roads: a real distinction Ergonomics, 33 (10-11) (1990), pp. 13151332 http://dx.doi.org/10.1080/00140139008925335

P.M. Salmon, G.J.M. Read, N.A. Stanton, M.G. Lenné The crash at Kerang: investigating systemic and psychological factors leading to unintentional non-compliance at rail level crossings Accid. Anal. Prev., 50 (0) (2013), pp. 1278-1288 http://dx.doi.org/10.1016/j.aap.2012.09.029

\section{A. Searle, L. Di Milia, D. Dawson An investigation of Risk Takers at Railway Level Crossings} CRC for Rail Innovation, Brisbane. QLD (2011)

S. Sposato, P. Bien-Aime, Chaudhary, M Public education and enforcement research study (DOT-VNTSC-FRA-06-03) U.S. Department of transportation Federal Railroad Administration (2006) 
S. Stewart, R. Brownlee, Stewart, D Second train warning at grade crossings. (TP 14,288E)

Transportation Development Centre (2004)

R. Werkman, S. Sjamaar, M. Coenders, G. van Meer, S. de Hek A combination of situational and generic approaches for studying and adressing level crossing accidents Paper presented at the Global Level Crossing and Trespass Symposium 2012, London, UK (2012)

L. Yardley, D. Marks Research Methods for Clinical and Health Psychology SAGE, London (2004) 